\title{
Can we estimate total magnetization directions from aeromagnetic data using Helbig's integrals?
}

\author{
Jeffrey D. Phillips \\ U.S. Geological Survey, Denver, Colorado 80225, U.S.A.
}

(Received January 30, 2004; Revised September 27, 2004; Accepted September 27, 2004)

\begin{abstract}
An algorithm that implements Helbig's (1963) integrals for estimating the vector components $\left(m_{x}, m_{y}, m_{z}\right)$ of the magnetic dipole moment from the first order moments of the vector magnetic field components $(\Delta X$, $\Delta Y, \Delta Z$ ) is tested on real and synthetic data. After a grid of total field aeromagnetic data is converted to vector component grids using Fourier filtering, Helbig's infinite integrals are evaluated as finite integrals in small moving windows using a quadrature algorithm based on the 2-D trapezoidal rule. Prior to integration, best-fit planar surfaces must be removed from the component data within the data windows in order to make the results independent of the coordinate system origin. Two different approaches are described for interpreting the results of the integration. In the "direct" method, results from pairs of different window sizes are compared to identify grid nodes where the angular difference between solutions is small. These solutions provide valid estimates of total magnetization directions for compact sources such as spheres or dipoles, but not for horizontally elongated or 2-D sources. In the "indirect" method, which is more forgiving of source geometry, results of the quadrature analysis are scanned for solutions that are parallel to a specified total magnetization direction.
\end{abstract}

Key words: Aeromagnetic, magnetization, interpretation.

\section{Introduction}

Total magnetization, which is the vector sum of the remanent magnetization and the induced magnetization, is the effective magnetization we see when looking at anomalies on an aeromagnetic map. Knowledge of total magnetization directions is useful for modeling the sources of magnetic anomalies, for filtering operations such as reductionto-the-pole or pseudogravity transformations where remanent magnetization cannot be ignored, and for inferring relative geologic ages or tectonic histories of the sources.

Traditional approaches to estimating the total magnetization direction for a 3-D source require that the shape of the body be known or assumed (Bhattacharyya, 1966; Emilia and Massey, 1974; Rao et al., 1977; Schnetzler and Taylor, 1984; Parker et al., 1987; Blakely, 1995, pp. 223-228). This requirement can be relaxed through an approach called "inversion of source moments" (Medeiros and Silva, 1995), which uses moments up to second order of a component of the magnetic field and involves an iterative solution. In general, these approaches are not appropriate for rapid analysis of large aeromagnetic data sets where the source geometries vary and source locations are not well known.

In a largely forgotten paper, Klaus Helbig (1963) developed integral relations for estimating the direction of total magnetization of a localized magnetic source body (or bodies) from first order moments of magnetic component data. In the late 1990's, Phillip Schmidt and David Clark of Australia's Commonwealth Scientific and Industrial Research

Published by TERRAPUB for The Society of Geomagnetism and Earth, Planetary and Space Sciences (SGEPSS), The Seismological Society of Japan, The Volcanological Society of Japan, The Geodetic Society of Japan, and The Japanese Society for Planetary Sciences.
Organisation (CSIRO) rediscovered Helbig's paper and advocated applying the method to modern total field data by using Fourier-method component transformations (Schmidt and Clark, 1997, 1998). They also presented some examples from Australia, one of which will be revisited here.

This paper describes a practical approach for estimating total magnetization directions from aeromagnetic data using Helbig's integrals. The approach involves evaluating Helbig's infinite integrals as finite integrals within small data windows of differing sizes, then either comparing the results from different window sizes to find grid nodes where the angular difference between magnetization solutions is small, or identifying grid nodes having magnetization directions close to a specified direction. The method is tested on several example data sets.

\section{Theory-Helbig's Integrals}

According to Helbig (1963), given magnetic field components $(\Delta X, \Delta Y, \Delta Z)$ produced by localized magnetic sources, all having the same total magnetization direction, the following integrals, which represent means and first order moments, should vanish:

$$
\begin{aligned}
& I_{1}=\int_{-\infty}^{\infty} \int_{-\infty}^{\infty} \Delta X d x d y=0 \\
& I_{2}=\int_{-\infty}^{\infty} \int_{-\infty}^{\infty} \Delta Y d x d y=0 \\
& I_{3}=\int_{-\infty}^{\infty} \int_{-\infty}^{\infty} \Delta Z d x d y=0 \\
& I_{4}=\int_{-\infty}^{\infty} \int_{-\infty}^{\infty} y \Delta X d x d y=0
\end{aligned}
$$




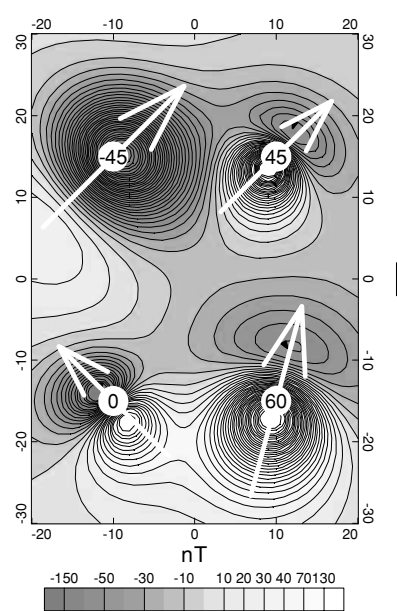

Total Field

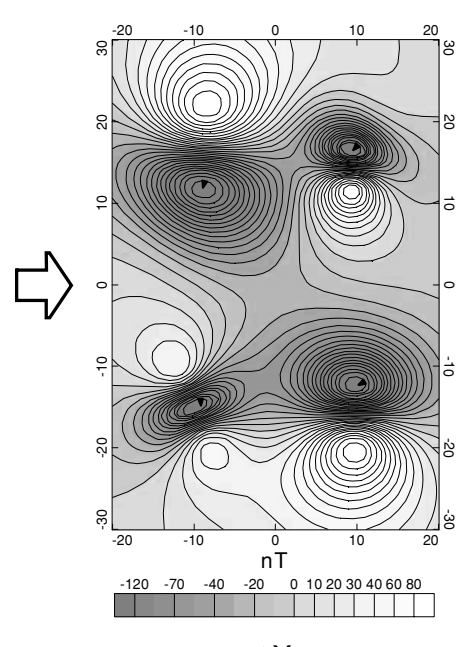

$\Delta \mathrm{X}$

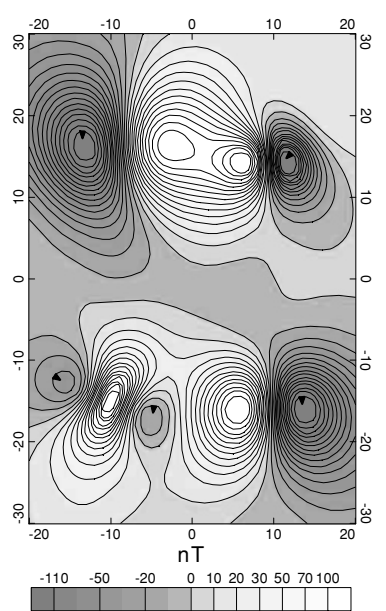

$\Delta \mathrm{Y}$

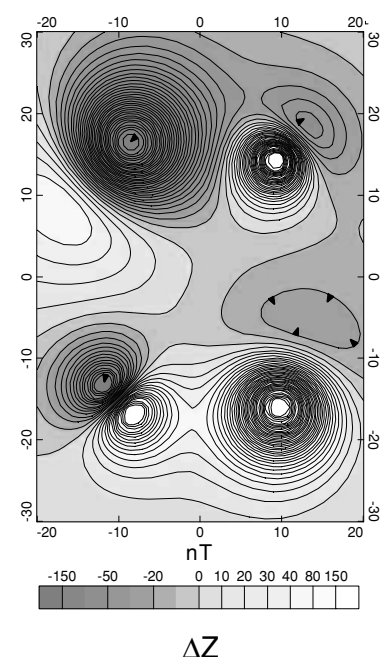

$\Delta \mathrm{Z}$

Fig. 1. The four-dipole example. The total field anomaly grid produced by four dipole sources having different magnetization directions (Total Field) is converted to magnetic component grids $(\Delta X, \Delta Y, \Delta Z)$ by applying Fourier filters. The arrows represent the horizontal components of the magnetizations, the filled circles indicate the horizontal source locations, and the numbers in the circles indicate the inclinations of the magnetizations. The regional field has an inclination of $60^{\circ}$ and a declination of $15^{\circ}$.

$$
I_{5}=\int_{-\infty}^{\infty} \int_{-\infty}^{\infty} x \Delta Y d x d y=0
$$

Furthermore (Helbig, 1963), the following integrals, representing the remaining first order moments, should yield the average components of the magnetic dipole moment of the sources:

$$
\begin{aligned}
& I_{6}=-\frac{1}{2 \pi} \int_{-\infty}^{\infty} \int_{-\infty}^{\infty} x \Delta X d x d y=m_{z} \\
& I_{7}=-\frac{1}{2 \pi} \int_{-\infty}^{\infty} \int_{-\infty}^{\infty} y \Delta Y d x d y=m_{z} \\
& I_{8}=-\frac{1}{2 \pi} \int_{-\infty}^{\infty} \int_{-\infty}^{\infty} x \Delta Z d x d y=m_{x} \\
& I_{9}=-\frac{1}{2 \pi} \int_{-\infty}^{\infty} \int_{-\infty}^{\infty} y \Delta Z d x d y=m_{y}
\end{aligned}
$$

Here negative signs have been added to correct Helbig's published equations for the case where the plane of integration is above the sources and $z$ is positive downward. Note that there are two estimates for the vertical component of the magnetic dipole moment, $m_{z}$.

Because the total magnetization is parallel to the magnetic dipole moment, it follows from (2) that the average magnetic dipole moment $|m|$, inclination $i_{m}$, and declination $d_{m}$ of the total magnetization are given by

$$
\begin{aligned}
|m| & =\sqrt{m_{x}^{2}+m_{y}^{2}+m_{z}^{2}}=\sqrt{I_{8}^{2}+I_{9}^{2}+\left(I_{6}+I_{7}\right)^{2} / 4} \\
i_{m} & =\arcsin \left[\frac{m_{z}}{|m|}\right]=\arcsin \left[\frac{\left(I_{6}+I_{7}\right)}{2|m|}\right]
\end{aligned}
$$

$$
d_{m}= \begin{cases}\arctan \left[\frac{m_{y}}{m_{x}}\right]=\arctan \left[\frac{I_{9}}{I_{8}}\right] & ; m_{x} \geq 0 \\ \arctan \left[\frac{m_{y}}{m_{x}}\right]+180^{\circ} & ; m_{x}<0, m_{y} \geq 0 \\ \arctan \left[\frac{m_{y}}{m_{x}}\right]-180^{\circ} & ; m_{x}<0, m_{y}<0\end{cases}
$$

With these principal values, $-90^{\circ} \leq i_{m} \leq 90^{\circ}$ and $-180^{\circ}$ $<d_{m} \leq 180^{\circ}$. By convention $x$ and $\Delta X$ are positive to the north, $y$ and $\Delta Y$ are positive to the east, $\Delta Z$ and $i_{m}$ are positive downward, and $d_{m}$ is positive clockwise from north when viewed from above.

\section{Implementation}

In order to apply Helbig's equations to modern total field aeromagnetic data, we must first convert the gridded magnetic data to vector component grids using Fourier filtering techniques (Lourenco and Morrison, 1973; Blakely, 1995, pp. 342-343). To do this, we only need to know the local geomagnetic field direction, which can be determined from models such as the International Geomagnetic Reference Field (IGRF, Macmillan et al., 2003). Theoretically such transformations should only be applied to data that are measured on, or continued to a horizontal surface, but in practice this restriction is often ignored. Figure 1 shows this transformation for a four-dipole example, in which each of the dipoles has a different magnetization direction. This four-dipole example will be used to illustrate the approach of this paper.

Helbig's four non-zero integrals ( $I_{6}$ through $\left.I_{9}\right)$ are evaluated from the component grids in sliding $(2 n+1) \times(2 n+1)$ windows using a 2-D quadrature algorithm, such as one based on the trapezoidal rule (McCracken and Dorn, 1964, pp. 191-192):

$$
\int_{x_{-n}}^{x_{n}} \int_{y_{-n}}^{y_{n}} f(x, y) d x d y \approx \frac{\Delta x \Delta y}{4} \sum_{i=-n}^{n} \sum_{j=-n}^{n} w_{i, j} f\left(x_{i}, y_{j}\right)
$$


Here $\Delta x$ and $\Delta y$ are the grid intervals, and the weights $w_{i, j}$ take on the following values:

$$
\begin{aligned}
& i=-n \quad i=n \\
& \begin{array}{llllllll}
j=n & 1 & 2 & 2 & \ldots & 2 & 2 & 1 \\
& 2 & 4 & 4 & \ldots & 4 & 4 & 2
\end{array} \\
& \begin{array}{lllllll}
2 & 4 & 4 & \ldots & 4 & 4 & 2
\end{array} \\
& \begin{array}{lllllll}
\vdots & \vdots & \vdots & \ddots & \vdots & \vdots & \vdots
\end{array} \\
& \begin{array}{lllllll}
2 & 4 & 4 & \ldots & 4 & 4 & 2
\end{array} \\
& j=-n \quad \begin{array}{llllllll} 
& 2 & 4 & 4 & \ldots & 4 & 4 & 2 \\
& 1 & 2 & 2 & \ldots & 2 & 2 & 1
\end{array}
\end{aligned}
$$

or

$$
w_{i, j}=\left\{\begin{array}{l}
4 \text { in window interior } \\
2 \text { on window edges } \\
1 \text { in window corners }
\end{array}\right.
$$

In order to approximately satisfy Helbig's vanishing integrals (1), it was found to be necessary to remove best-fit planar surfaces from $\Delta X, \Delta Y$, and $\Delta Z$ within each small data window before integration. If (1) is not satisfied, the non-zero moments (2) will depend on the choice of origin. For example, if $x^{\prime}=x+x_{0}$,

$$
\begin{aligned}
I_{6} & =\frac{1}{2 \pi} \int_{-\infty}^{\infty} \int_{-\infty}^{\infty} x^{\prime} \Delta X d x^{\prime} d y \\
& =\frac{1}{2 \pi} \int_{-\infty}^{\infty} \int_{-\infty}^{\infty}\left(x+x_{0}\right) \Delta X d x d y \\
& =\frac{1}{2 \pi} \int_{-\infty}^{\infty} \int_{-\infty}^{\infty} x \Delta X d x d y+\frac{x_{0}}{2 \pi} \int_{-\infty}^{\infty} \int_{-\infty}^{\infty} \Delta X d x d y \\
& =I_{6}+\frac{x_{0}}{2 \pi} I_{1}
\end{aligned}
$$

which can only be satisfied if $I_{1}=0$. Removing a mean value from $\Delta X, \Delta Y$, and $\Delta Z$ within each window will assure that $I_{1}, I_{2}$, and $I_{3}$ are near zero. Removing a bestfit planar surface within each window will help reduce the magnitudes of $I_{4}$ and $I_{5}$ (Fig. 2).

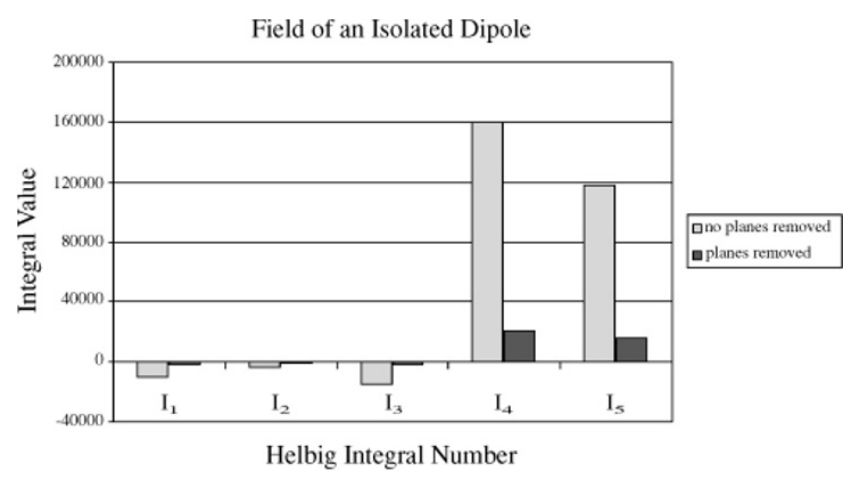

Fig. 2. Chart showing how removing planar surfaces from the component grids prior to integration reduces the estimated magnitudes of Helbig's vanishing integrals $\left(I_{1}\right.$ through $\left.I_{5}\right)$ for the field of an isolated dipole . Integrals $I_{1}$ through $I_{3}$ are not completely reduced to zero due to the trapezoidal weighting used in the quadrature algorithm.

\section{$13 \times 13$}
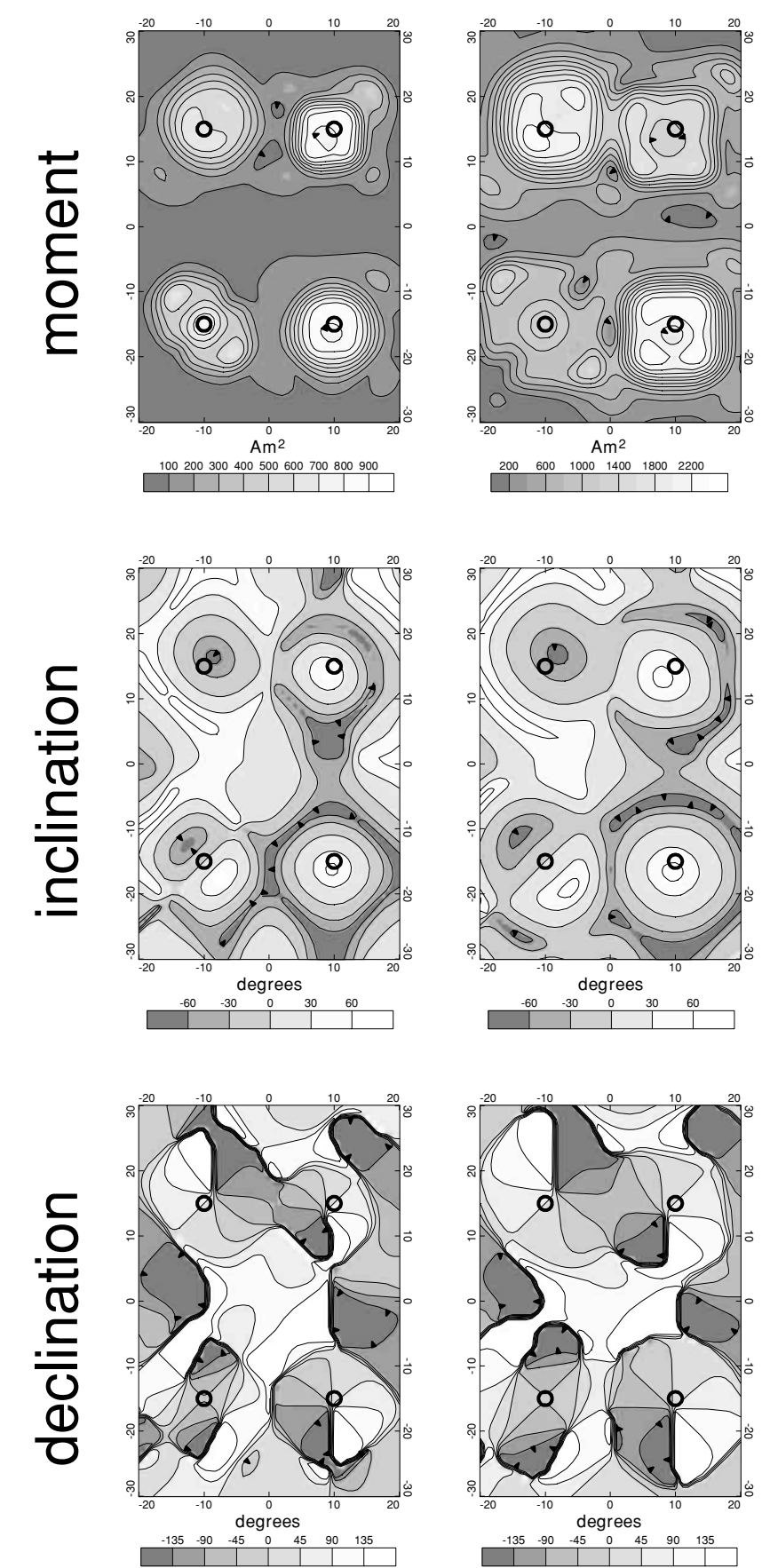

Fig. 3. Grids of moment, inclination, and declination of the magnetization for the four-dipole example as estimated using a $13 \times 13$ window and a $19 \times 19$ window. At the locations of the dipoles, shown by the black circles, the inclinations and declinations are correctly estimated.

\section{Two Methods}

The results of the quadrature analysis are grids of magnetic moment, inclination, and declination for each of the chosen window sizes. These grids must be analyzed to identify the grid nodes containing the best solutions - those associated with the actual magnetic sources. Two approaches have been tested. In the first approach, we try to directly estimate the valid magnetization directions (and source locations) by identifying grid nodes where the quadrature results 
from different window sizes are in close angular agreement. This approach will be called the "direct" method, and is further subdivided into "basic" and "extended" versions. In the second approach, we seek grid nodes having solutions close to a specified magnetization direction, such as the inducing field direction. This will be called the "indirect" method.

\subsection{The basic "direct" method}

The basic "direct" method involves evaluating Helbig's integrals using two different window sizes, finding the grid nodes where the angular differences between the two solutions are small, and calling these the best solutions. The window sizes should be chosen to encompass representative isolated anomalies. Figure 3 shows grids of moment, incli-

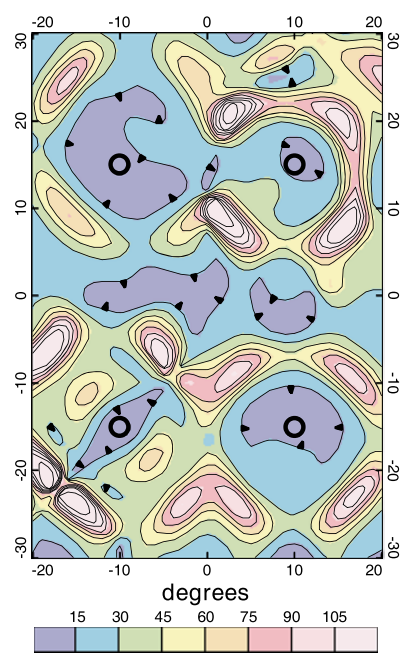

(a)

angular

difference

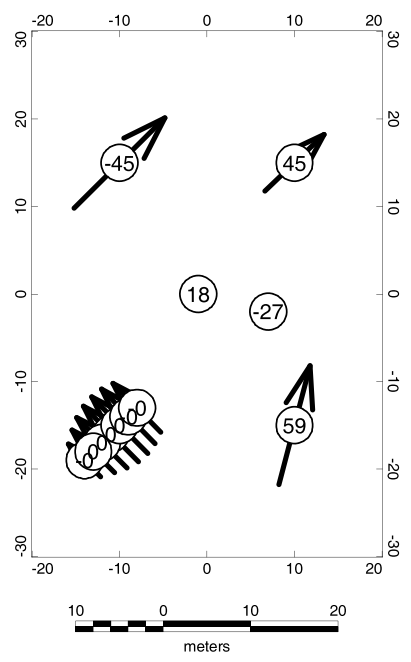

(c)

smallest angular differences

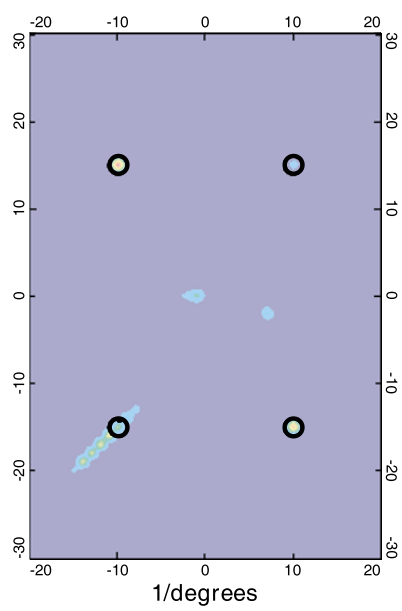

(b)

reciprocal of angular difference

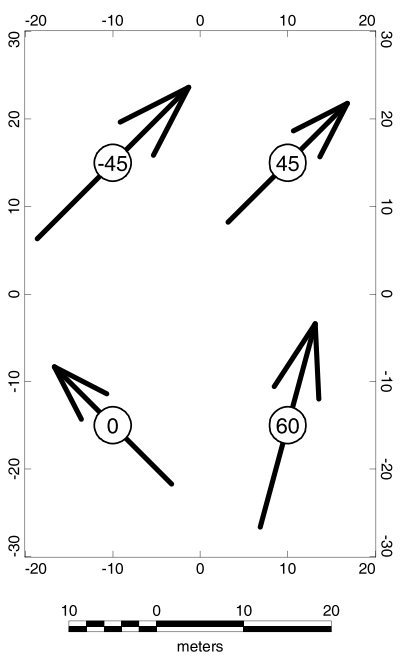

(d) answer

Fig. 4. The basic "direct" method applied to the four-dipole example. (a) Angular difference between the results of the $13 \times 13$ window and the $19 \times 19$ window. Black circles indicate the dipole locations. (b) The reciprocal of the angular difference. (c) The magnetization solutions at the twelve grid nodes having the smallest angular differences. Symbols are as in Fig. 1. (d) The correct magnetizations.

nation, and declination of the magnetization as estimated using a 13 by 13 window and a 19 by 19 window on the four-dipole example, which has a grid cell size of 1 meter. Odd window sizes are used so the solutions can be assigned to the grid node at the center of the window. Overlapping windows provide solutions at all grid nodes, except near the edges of the data coverage. At the locations of the dipoles, shown by the black circles, the inclinations and declinations are estimated correctly. However, if the center of the window is moved even slightly away from a dipole location, the estimated directions can be grossly inaccurate. Note that the estimated moments increase in magnitude as the window size increases.

Figure 4(a) shows the angular difference between the two solutions. Note that the difference is always low at the dipole locations. To emphasize this, Fig. 4(b) shows the reciprocal of the angular difference; the dipole locations are apparent. The southwest dipole is poorly located, probably because its magnetization is at approximately right angles to the regional field direction. The apparent solutions in the center of the area can be ignored, because they have very small moments.

Figure 4(c) shows the twelve solutions for the four-dipole example having the smallest angular differences. The correct answer is in Fig. 4(d). Can we use Helbig's integrals to estimate total magnetization directions? Figure 4 suggests that at least for semi-isolated dipoles the answer is "yes".

\subsection{The extended "direct" method}

In an attempt to minimize the number of extraneous solutions, an extended version of the direct method was developed. This involves generating solutions for many different pairs of window sizes using the basic direct method, and looking for clustering in the results. A cluster indicates repeated solutions from different pairs of window sizes. For example, by using 12 odd window sizes varying from 3 by 3 grid nodes to 25 by 25 grid nodes, one can get 66 different pairs of window sizes as indicated by the circles in Fig. 5, leading to 66 different basic direct solutions.

The maximum allowable angular difference between

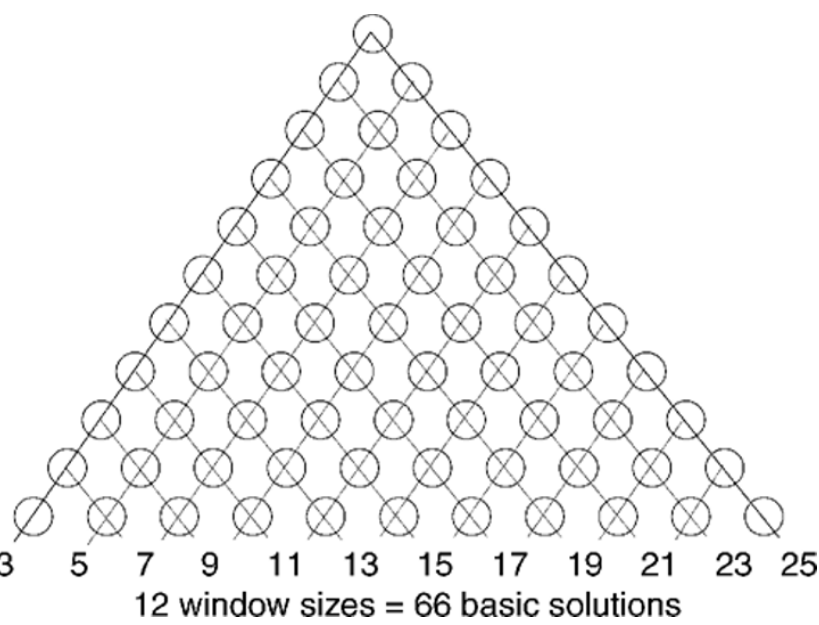

Fig. 5. The extended direct method involves applying the basic direct method to many different pairs of window sizes. The circles represent the 66 different basic direct solutions that result from combinations of the twelve odd window sizes ranging from $3 \times 3$ to $25 \times 25$ grid nodes. 
pairs of solutions must be adjusted for the difference in the size of the windows. The smallest maximum angular difference is specified for solutions where the edges of the two windows differ by only two grid nodes (one "lag"). The allowable angular difference increases linearly as the difference in window size increases, so that the maximum allowable angular difference between solutions from a $3 \times 3$ and a $25 \times 25$ window (eleven "lags") is 11 times the maximum angular difference between a $3 \times 3$ and a $5 \times 5$ window. This linear relationship was chosen to approximately equalize the number of basic direct solutions for each pair of window sizes.

The estimated magnetic moment is also a function of window size, so the magnetic moments of the basic direct solutions are all normalized to the range of the moment

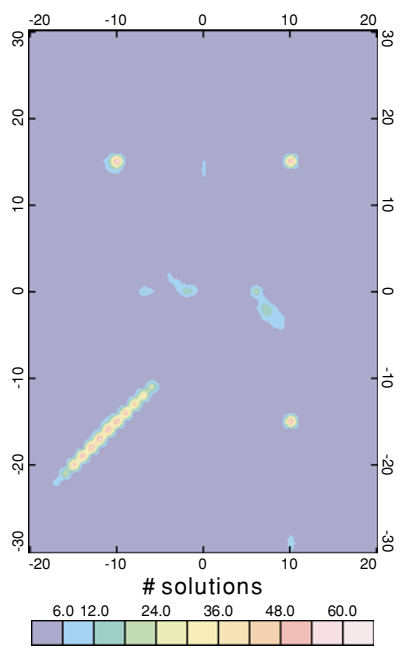

(a)

number of solutions at each grid node

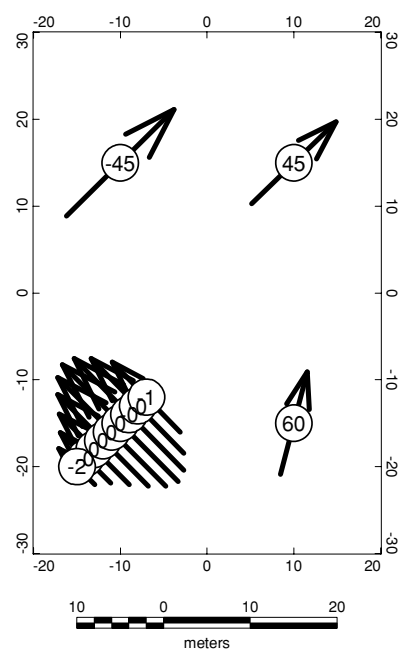

(b) averaged results

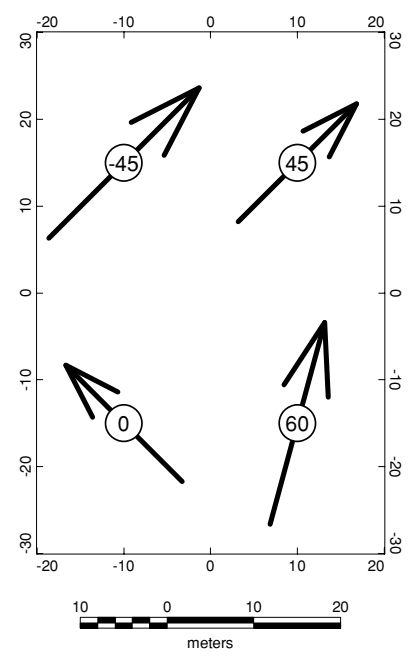

(c) correct answer

Fig. 6. Application of the extended direct method to the four-dipole example. (a) Number of basic direct solutions at each grid node having angular differences of one degree per lag or less. Any grid node can have from 0 to 66 solutions. (b) Averaged magnetization solutions for grid nodes having 40 or more solutions. (c) The correct magnetizations. The extended approach has removed the less reliable solutions of Fig. 4.

for the largest window at the end of processing. The windowed approach almost certainly provides poor estimates of the magnetic moments, even after this normalization. So the resulting magnetic moments should be considered relative values rather than absolute values. In contrast, the

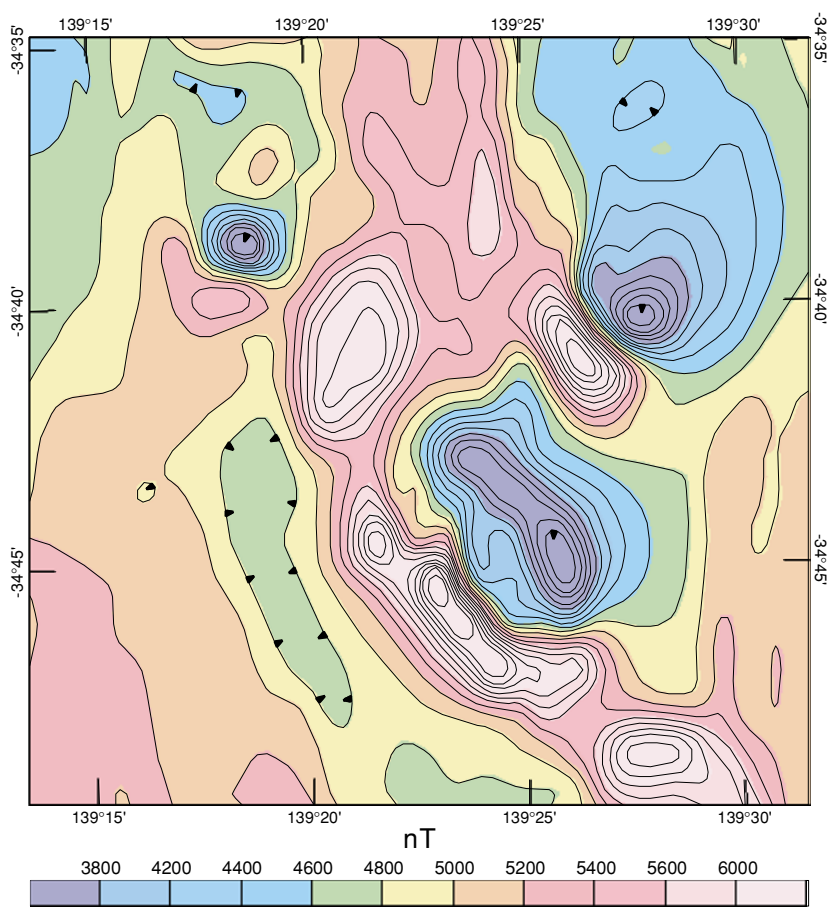

(a)

Aeromagnetic Map

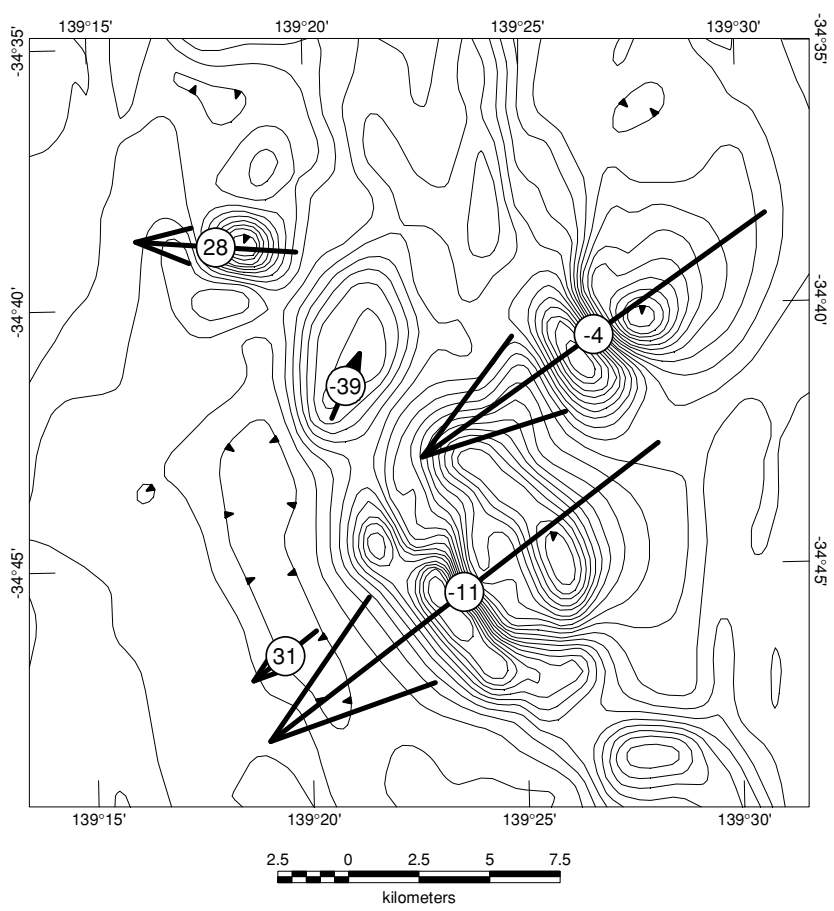

(b)

Helbig Solution

Fig. 7. The Black Hill Norite of South Australia is associated with the magnetic low in the northeast quadrant of the aeromagnetic map (a). A similar low appears to the southwest. The results of the extended direct method are shown in (b) and in Table 1. Aeromagnetic data (c) Commonwealth of Australia (Geoscience Australia) 2003. 
Table 1. Measured and estimated total magnetization directions for the Black Hill Norite, South Australia.

\begin{tabular}{lccc}
\hline & inclination & declination & error \\
\hline Measured Values & & & \\
Rajagopalan et al. $(1993)$ & $-20^{\circ}$ & $-131^{\circ}$ & \\
\hline Estimated Values & & & \\
Schmidt and Clark (1997) & $-32^{\circ}$ & $-151^{\circ}$ & $22^{\circ}$ \\
This Study: NE body & $-4^{\circ}$ & $-126^{\circ}$ & $17^{\circ}$ \\
This Study: SW body & $-11^{\circ}$ & $-128^{\circ}$ & $10^{\circ}$ \\
\hline
\end{tabular}

estimated inclinations and declinations are independent of window size and represent robust estimates of the total magnetization direction.

Figure 6 shows the results of applying the extended direct method to the four-dipole example using the window sizes of Fig. 5. Any grid node can have from 0 to 66 solutions (Fig. 6(a)). The solutions are concentrated at the dipole locations. By averaging solutions for grid nodes having 40 or more solutions (Fig. 6(b)), the extended direct method has succeeded in eliminating the less reliable solutions in the center of the area (Fig. 4).

\subsubsection{Example-Black Hill Norite, South Australia} Figure 7(a) shows a real-world example where the direct method works. This example was used by Schmidt and Clark (1997) for their Helbig analysis, and here an attempt has been made to reproduce their results. The Black Hill Norite is associated with the magnetic low in the northeast quadrant of the aeromagnetic map. A similar low appears $8.5 \mathrm{~km}$ to the southwest. The results of the extended direct method are shown in Fig. 7(b). Two bodies have large total magnetizations with southwest declinations and shallow upward directed inclinations. The results of the analysis can vary by a few degrees depending on how the clustering is

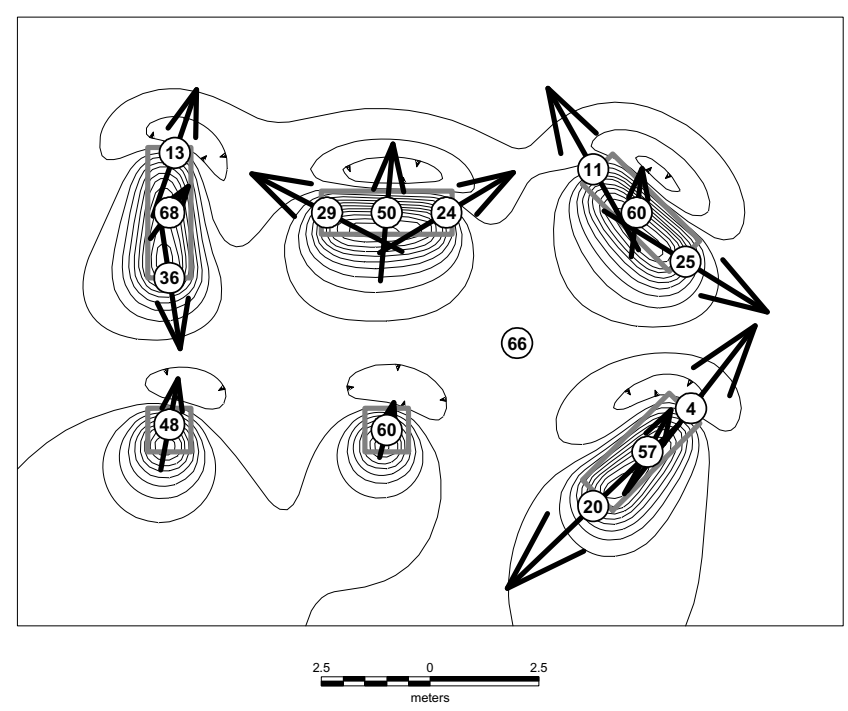

Fig. 8. Extended direct method results for a model consisting of six prisms, showing how horizontally elongated sources produce results that are accurate near their centers of mass (centers of dipole moment), but distorted near their ends. Basic direct method solutions were averaged at grid cells containing at least nine solutions; these averaged solutions were then clustered within a radius of 0.75 meters, three times the grid interval. done. Here basic direct solutions that agreed to within one degree per lag were averaged at grid nodes containing at least five solutions. These averaged values were then clustered using a radius of 5 kilometers. The grid interval was 400 meters.

Table 1 shows that the measured average total magnetization direction for the Black Hill Norite is $-20^{\circ}$ inclination and $-131^{\circ}$ declination (Rajagopalan et al., 1993). Schmidt and Clark's (1997) Helbig analysis resulted in a direction within $22^{\circ}$ of the measured value. Results of the present study are even closer to the measured value (within $17^{\circ}$ and $10^{\circ}$ for the two bodies).

\subsection{Elongated sources-a limitation of the "direct" method}

When the source bodies become elongated in the horizontal direction, the direct method starts to break down. Figure 8 shows extended direct method results for a model consisting of six prisms, five of which have dimensions of $1 \times 1 \times 3$ meters, and one, in the bottom center, is a 1 -meter cube. All prisms are magnetized in the direction of the geomagnetic field, inclination $60^{\circ}$, declination $15^{\circ}$. Only the cube and the vertically elongated prism (lower left) give reasonable results. The horizontally elongated prisms produce results that are accurate near their centers of mass (or centers of dipole moment), but distorted near their ends.

In the extreme case, where the magnetic bodies are so large or so elongated that any finite window will only see the body edges, the direct method will predict magnetizations that are perpendicular to the body edges, as is illustrated by a synthetic example of a pipeline over a buried fault (Fig. 9). Here the true magnetization direction is north. These results are not surprising. They are reminding us that for 2-D sources, we can really only estimate the component of the magnetization that is perpendicular to the strike direction.

In summary, the direct method allows the total magnetization direction to be estimated from Helbig's integrals without any a priori knowledge. But, it requires semiisolated, compact source bodies without significant horizontal elongation, such as dipoles, spheres, or vertical cylinders.

\subsection{The indirect method}

Where the directs method fails, the Helbig integral results can still be used to search for sources having a specified total magnetization direction, such as the inducing field direction, a measured total magnetization direction, or an assumed direction based on geologic age. This approach, which will be called the "indirect" method, simply involves 


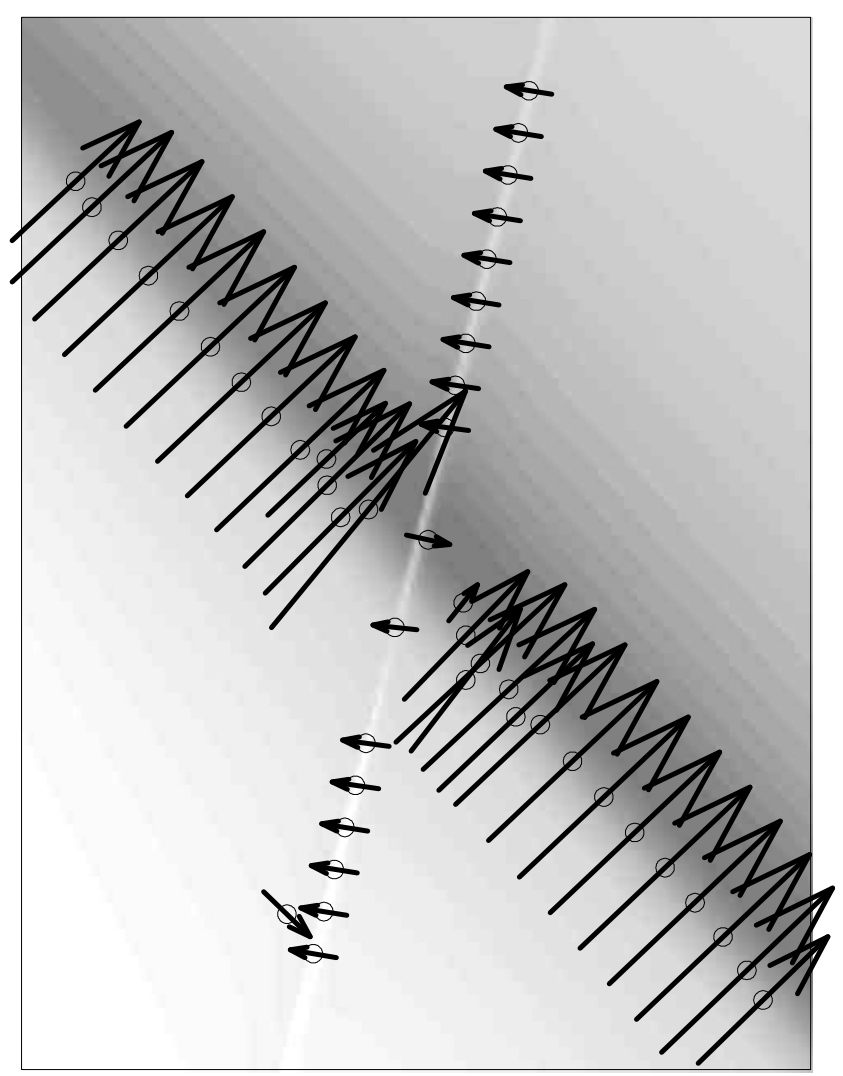

nT

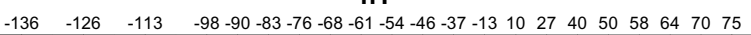

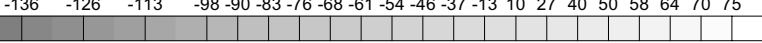

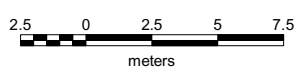

Fig. 9. Extended direct method results (circles and arrows) for a model consisting of a magnetic field (graytone image) produced by a north-northeast-trending pipeline over a buried southeast-striking fault. For this extreme case of elongated magnetic source bodies, the method predicts magnetizations that are perpendicular to the body edges, because for 2-D sources only the component of the magnetization that is perpendicular to the strike direction can be estimated. The true magnetization direction is north. In this example, the maximum angular difference between lag-one solutions was taken to be $0.5^{\circ}$, and a minimum magnetic moment of 2 ampere-meter ${ }^{2}$ was used to reduce noise. Results were clustered using a radius of 1 meter ( 5 grid intervals) and a minimum cluster size of two solutions.

identifying the grid nodes from the quadrature results where the estimated magnetization direction is close to a specified direction. If we again use twelve different window sizes for the quadrature analysis, then there will be from zero to twelve indirect-method solutions at each grid node. These solutions, like the direct solutions, can be thresholded, averaged, and (or) clustered to achieve a satisfactory result.

The goal of the indirect method is to identify the centers of mass (or centers of dipole moment) of sources that may have magnetization directions parallel to the specified direction. This goal is clearly met for the elongated prism example (Fig. 10), when the specified magnetization direction is chosen to be the inducing field direction. A comparison of Figs. 10 and 8 shows that the indirect method is much less sensitive to elongation of the sources than is the direct method.

In a second example, two likely sources of Black Hill

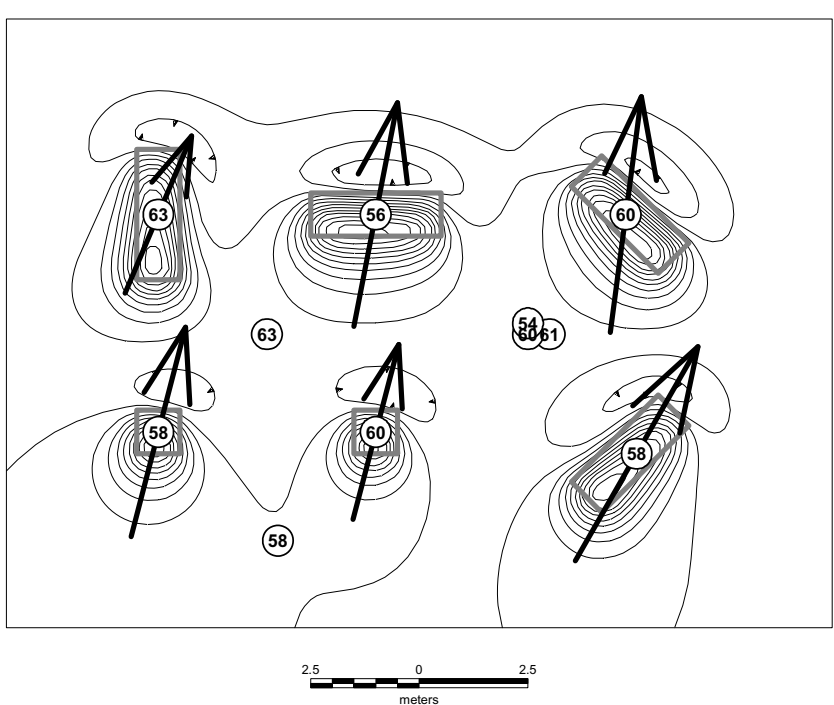

Fig. 10. Results of searching for all solutions within ten degrees of the inducing field direction for the elongated prism example. With twelve window sizes, each grid node can have up to twelve solutions. The results have been averaged at grid nodes having at least five solutions.

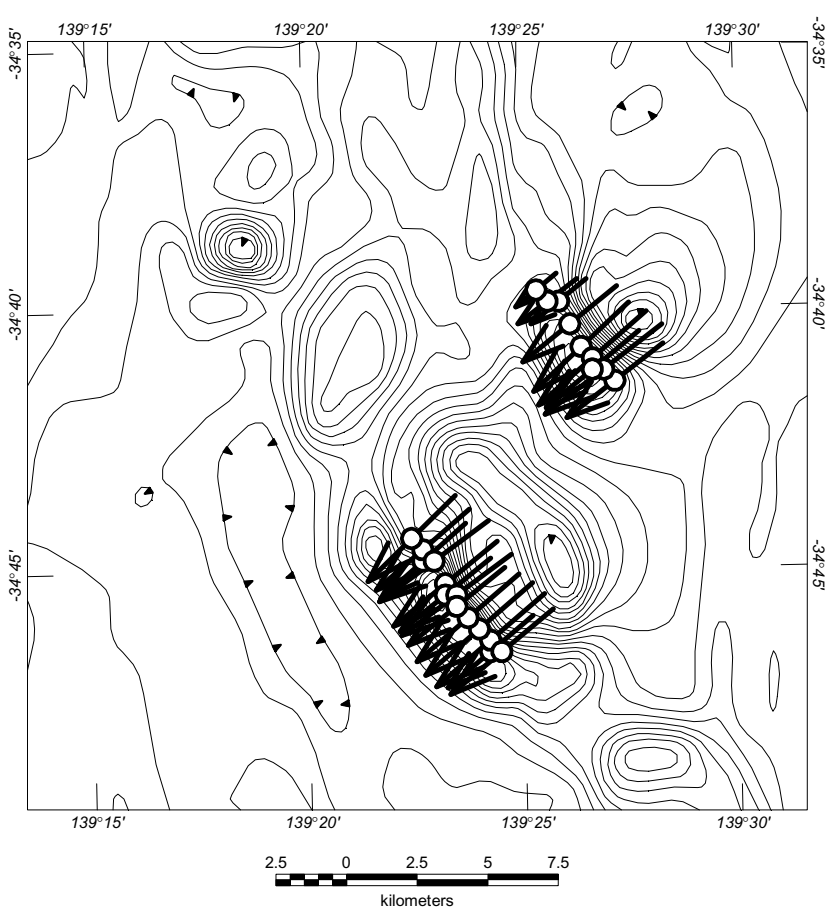

Fig. 11. Results of searching for all solutions within ten degrees of the measured total magnetization direction of the Black Hill Norite. Again only grid nodes having at least five of the twelve possible solutions are shown. Aeromagnetic data (C)Commonwealth of Australia (Geoscience Australia) 2003.

Norite are identified from the aeromagnetic data (Fig. 11) by searching for sources having the measured total magnetization direction for this unit. A final example of indirect-method results involves aeromagnetic data (USGS and Sander Geophysics, Ltd., 1998) over a Cenozoic volcanic center in New Mexico (Fig. 12). Volcanic sources having normal and reversed magnetizations are identified by searching for magnetization directions parallel to, and antiparallel to the current regional field direction, which is 


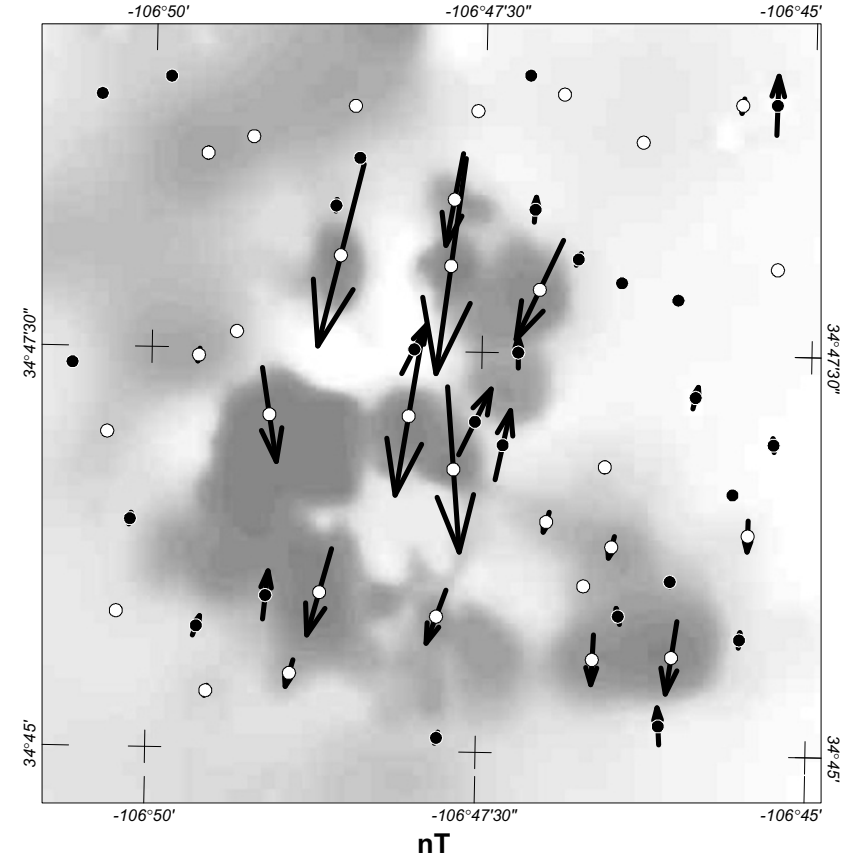

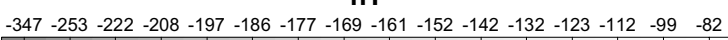

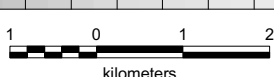

Fig. 12. Indirect method results for a Cenozoic volcanic center in the southern Albuquerque basin, New Mexico. Because the flows are Cenozoic, we know that the magnetizations are approximately parallel or anti-parallel to the present day geomagnetic field. Solutions that fall within 20 degrees of the geomagnetic field direction are shown, with white-filled symbols and south-directed arrows indicating reversed magnetizations and black-filled symbols and north-directed arrows indicating normal magnetizations. Clustering has been used to reduce the number of solutions.

close to the Cenozoic direction.

This last example points out a possible ambiguity in the method. The reversely magnetized solutions are probably due to actual reversely magnetized rocks, but they could be due to zones of weaker normal magnetization within an otherwise strongly magnetized rock mass of normal polarity. Where the country rocks are strongly magnetized, it is important to remember that the magnetic anomalies are produced by magnetization contrasts rather than absolute magnetizations, so the estimated magnetization directions represent the contrasts (Schmidt and Clark, 1998).

\section{Conclusions}

The Helbig integrals can be evaluated by quadrature analysis in small data windows on magnetic component grids derived from total field magnetic anomaly data. Best-fit planar surfaces must be removed from the component data in each small data window prior to integration. The windowed results provide local estimates of the moment, inclination, and declination of the total magnetization, which are valid near the centers of mass (centers of dipole moment) of the sources. By evaluating the integrals using several different window sizes, then comparing results from pairs of window sizes to find the grid nodes where the solutions are in close angular agreement, the source locations and the valid total magnetization directions can be identified. This "direct" ap- proach works best for isolated, compact sources, and largely fails for horizontally elongated sources. An "indirect" approach, in which the results from the different window sizes are searched to identify grid nodes where the estimated total magnetization is close to a specified direction, can always be used to locate sources having that magnetization direction. In both the "direct" and "indirect" methods the scatter in solutions is reduced by specifying a minimum number of solutions per grid node, averaging the solutions at grid nodes containing this minimum number, and optionally clustering the averaged results within some radius.

Application of Helbig's integrals should prove useful for rapid analysis of gridded aeromagnetic and ground magnetic anomaly data. The "direct" method should be particularly valuable for locating the centers of mass (centers of dipole moment) and in characterizing the total magnetization directions of both localized geologic bodies such as small plutons, volcanic centers, and kimberlite pipes, and localized cultural sources such as well casings, buried drums, and unexploded ordnance.

The "indirect" method should be useful for locating the centers of mass (centers of dipole moment) of magnetic sources having a specified total magnetization direction. For example, by specifying the inducing field direction, bodies having predominantly induced magnetization can be located, and, by exclusion, bodies having significant remanant magnetization can be identified. By specifying a measured total magnetization direction, multiple bodies having the same geologic age and magnetic properties can be identified (e.g., the Black Hill Norite). Source bodies having primarily remanent magnetization, such as volcanic flows, can be located by specifying normal and reversed magnetization directions for rocks of that particular geologic age.

Acknowledgments. This paper was presented at the 2003 International Union of Geodesy and Geophysics (IUGG) meeting in Sapporo, Japan, and it incorporates aeromagnetic data which is: (C)Commonwealth of Australia (Geoscience Australia) 2003. The author is grateful to Phillip Schmidt and David Clark for rediscovering Helbig's paper and demonstrating the practical value of his equations through published results on Australian aeromagnetic data. A discussion with Dr. Schmidt at his 2002 European Geophysical Society poster presentation prompted the research that led to this paper. The author also thanks Rick Saltus and Bruce Smith for thoughtful reviews of an early draft, Rick Blakely and David Clark for helpful reviews of the submitted manuscript, and Yaoguo $\mathrm{Li}$ for pointing out the sign error in Helbig's published integrals.

\section{References}

Bhattacharyya, B. K., A method for computing the total magnetization vector and the dimensions of a rectangular block-shaped body from magnetic anomalies, Geophysics, 31, 74-96, 1966.

Blakely, R. J., Potential Theory in Gravity and Magnetic Applications, Cambridge University Press, Cambridge, 441 pp., 1995.

Emilia, D. A. and R. L. Massey, Magnetization estimation for nonuniformly magnetized seamounts, Geophysics, 39, 223-321, 1974.

Helbig, K., Some integrals of magnetic anomalies and their relation to the parameters of the disturbing body, Zeitschrift für Geophysik, 29(2), 8396, 1963.

Lourenco, J. S. and H. F. Morrison, Vector magnetic anomalies derived from measurements of a single component of the field, Geophysics, 38(2), 359-368, 1973.

Macmillian, S., S. Maus, T. Bondar, A. Chambodut, V. Golovkov, R. Holme, B. Langlais, V. Lesur, F. Lowes, H. Lühr, W. Mai, M. Mandea, N. Olsen, M. Rother, T. Sabaka, A. Thomson, and I. Wardinski, Ninth 
generation International Geomagnetic Reference Field released, EOS, Transactions, American Geophysical Union, 84(46), 503, 18 November 2003.

McCracken, D. D. and W. S. Dorn, Numerical Methods and Fortran Programming, John Wiley and Sons, Inc., New York, 457 pp., 1964.

Mederios, W. E. and J. B. C. Silva, Simultaneous estimation of total magnetization direction and 3-D spatial orientation, Geophysics, 60(5), 1365-1377, 1995.

Parker, R. L., L. Shure, and J. A. Hildebrand, The application of inverse theory to seamount magnetism, Rev. Geophys., 25, 17-40, 1987.

Rajagopalan, S., P. Schmidt, and D. Clark, Rock magnetism and geophysical interpretation of the Black Hill Norite, South Australia, Exploration Geophysics, 24, 209-212, 1993.

Rao, B. S. R., T. K. S. Prakasa Rao, and A. S. Krishna Murthy, A note on magnetized spheres, Geophys. Prosp., 25, 746-757, 1977.
Schmidt, P. W. and D. A. Clark, Directions of magnetization and vector anomalies derived from total field surveys, Preview, 70, 30-32, 1997.

Schmidt, P. W. and D. A. Clark, The calculation of magnetic components and moments from TMI: A case history from the Tuckers igneous complex, Queensland, Exploration Geophysics, 29, 609-614, 1998.

Schnetzler, C. C. and P. T. Taylor, Evaluation of an observational method for estimation of remanent magnetization, Geophysics, 49, 282-290, 1984.

U.S. Geological Survey and Sander Geophysics, Ltd., Digital data from the Isleta-Kirtland aeromagnetic survey, collected south of Albuquerque, New Mexico, U.S. Geological Survey Open-File Report 98-341 (CDROM), 1998.

J. Phillips (e-mail: jeff@usgs.gov) 